\title{
Philonsorbonne
}

5 | 2011

Année 2010-2011

\section{Les mathématiques chez Musil et Wittgenstein : de la mesure des possibilités à leur invention}

Pierre FASULA

\section{(2) OpenEdition}

1 Journals

Édition électronique

URL : https://journals.openedition.org/philonsorbonne/310

DOI : 10.4000/philonsorbonne.310

ISSN : 2270-7336

Éditeur

Publications de la Sorbonne

Édition imprimée

Date de publication : 15 mai 2011

Pagination : 9-21

ISBN : 978-2-85944-680-2

ISSN : 1255-183X

Référence électronique

Pierre FASULA, «Les mathématiques chez Musil et Wittgenstein : de la mesure des possibilités à leur invention », Philonsorbonne [En ligne], 5 | 2011, mis en ligne le 03 février 2013, consulté le 08 juin 2021. URL : http://journals.openedition.org/philonsorbonne/310 ; DOI : https://doi.org/10.4000/

philonsorbonne.310

Ce document a été généré automatiquement le 8 juin 2021.

(c) Tous droits réservés 


\title{
Les mathématiques chez Musil et Wittgenstein : de la mesure des possibilités à leur invention
}

\author{
Pierre FASULA
}

1 Au tout début de L'Homme sans qualités, Robert Musil attribue à Ulrich, le personnage principal, un sens du possible qui n'est pas sans rappeler certains traits de la pratique wittgensteinienne de la philosophie :

L'homme qui en est doué, par exemple, ne dira pas: ici s'est produite, va se produire, doit se produire telle ou telle chose; mais il imaginera: ici pourrait, devrait se produire telle ou telle chose ; et quand on lui dit d'une chose qu'elle est comme elle est, il pense qu'elle pourrait aussi bien être autre. Ainsi pourrait-on définir simplement le sens du possible comme la faculté de penser tout ce qui pourrait être «aussi bien", et de ne pas accorder plus d'importance à ce qui est qu'à ce qui n'est pas ${ }^{1}$.

On peut alors penser à l'importance accordée par Ludwig Wittgenstein à l'imagination dans la pratique de la philosophie, mais aussi à sa réflexion sur les variations d'aspects et à leur usage, ainsi qu'à un point de vue logique qui ne favorise pas plus ce qui est le cas que ce qui n'est pas le cas. Or, à chaque fois, ce sont là autant d'éléments d'une méthode où la notion de possibilité joue un rôle central.

Dans le cadre de cet article, on ne cherchera pourtant pas à développer cette comparaison sur le plan de la philosophie, mais plutôt à déplacer ce thème du sens du possible du côté des mathématiques, puisque c'est en bonne partie sur ce terrain que les deux auteurs ont développé une pensée originale de la possibilité. On sait en effet que Robert Musil a de son côté accordé une grande importance aux probabilités, notamment dans leur application à l'histoire, la société et l'homme ${ }^{2}$, alors que Ludwig Wittgenstein s'est davantage penché sur le rôle des mathématiques dans la détermination de ce qui est possible ou pas $^{3}$. Il faut cependant préciser que ni Robert Musil ni Ludwig Wittgenstein n'ont cantonné leur réflexion à l'un ou l'autre aspect des mathématiques, si bien que l'intérêt pour la mesure de la probabilité et la détermination de nouvelles possibilités se retrouve dans chaque œuvre. Tout le 
problème est alors de savoir, d'une part, comment ces deux aspects s'articulent, et d'autre part, dans quelle mesure la comparaison entre ces deux auteurs peut être poursuivie jusque dans le domaine des mathématiques.

\section{La mesure de la probabilité et le rôle des fréquences dans sa définition}

4 Une manière de comprendre les positions respectives de Ludwig Wittgenstein et de Robert Musil, concernant la probabilité, est de les rapporter à leurs tenants et à leurs aboutissants, pour les situer dans la cartographie des réflexions sur ce sujet.

Si l'on regarde du côté des tenants, on trouve pour Ludwig Wittgenstein, ou en tout cas pour son Tractatus, la Wissenschaftslehre de Bernard Bolzano, au point que Georg Henrik Von Wright peut affirmer : "Il est une définition de la probabilité qui répond sur tous les points essentiels à celle de Wittgenstein ; c'est celle qui a été proposée, il y a presque un siècle, par Bolzano dans sa Wissenschaftslehre de 1837. [...] Il semble approprié de parler d'une seule et même définition de la probabilité que l'on appellera la définition bolzano-wittgensteinienne $»^{4}$, définition qui est de nature logique. Pour Musil, dans la liste que l'on trouve dans ses Journaux ${ }^{5}$ et qui contient plus d'une trentaine de titres sur le sujet, c'est plutôt le livre de Timerding, Die Analyse des Zufalls ${ }^{6}$, qui a compté, dans sa défense prudente d'une conception statistique des probabilités. Quand on regarde ces lectures, on voit donc se profiler déjà à l'arrière-plan l'opposition classique entre la conception logique et la conception statistique de la probabilité.

Or, cette différence se retrouve aussi parmi les interlocuteurs de nos deux auteurs. À la suite de son maître, Friedrich Waismann s'est fait le défenseur de la conception logique de la probabilité :

Le but des discussions qui suivent est la clarification logique du concept de probabilité. Elles veulent donner une réponse déterminée à la question de savoir ce que signifie la probabilité et ce qu'est le sens des énoncés de probabilités. En accord avec Leibniz et Bolzano, je crois que la théorie de la probabilité est une branche de la logique. Et je veux exposer ici comment cette conception peut, par l'utilisation des pensées de Wittgenstein, être libérée des difficultés qui jusqu'à présent entravaient son acceptation ${ }^{7}$.

7 Pour ce faire, il s'attaque à deux conceptions de la probabilité, la deuxième étant celle de Richard Von Mises, qui défendait une conception fréquentiste de la probabilité et s'intéressait plus généralement à ses applications possibles à la réalité. Or, Richard Von Mises est justement devenu un interlocuteur de Robert Musil, quand celui-ci s'est installé à Berlin dans les années 1930-1933. Et il est tout à fait vraisemblable que Robert Musil se soit accordé avec lui non seulement à propos de Rainer Maria Rilke, dont Richard Von Mises collectionnait les textes, mais aussi à propos de cette défense de la conception fréquentiste des probabilités.

C'est sur ce fond que l'on peut alors apprécier la différence entre Ludwig Wittgenstein et Robert Musil dans leurs définitions respectives des probabilités. Le premier introduit sa définition des probabilités à un moment du Tractatus où il rend compte des rapports entre propositions au moyen de leurs raisons de vérité. Les probabilités sont donc définies ainsi :

$5.15-\mathrm{Si} \mathrm{V}_{\mathrm{r}}$ est le nombre de fondements de vérité de la proposition « $\mathrm{r} », \mathrm{~V}_{\mathrm{rs}}$ le nombre des fondements de vérité de la proposition « $\mathrm{s}$ » qui sont en même temps 
fondements de vérité de « $\mathrm{r}$ », nous nommons alors le rapport $\mathrm{V}_{\mathrm{rs}}: \mathrm{V}_{\mathrm{r}}$ mesure de la probabilité que la proposition « $\mathrm{r} »$ confère à la proposition « $\mathrm{S} »^{8}$.

On parlera donc d'une définition logique dans la mesure où la probabilité n'est pas la qualité d'un événement mais un rapport entre deux propositions et plus précisément entre leurs raisons de vérité : «5.1511 - Il n’y a pas d'objet particulier propre aux propositions de probabilité. [...] 5.156 - [...] La proposition de probabilité est comme un extrait d'autres propositions $"{ }^{9}$.

Le second introduit les probabilités d'une tout autre manière, par exemple dans les réflexions d'Ulrich sur l'homme moyen, lors de ses promenades dans la foule avec sa sœur Agathe :

Dans ces pensées se glissait cependant aussi le souvenir du calcul des moyennes tel qu'on l'entend dans le calcul des probabilités. Avec une sérénité froide et presque indécente, les règles de la probabilité se fondent sur le fait que les événements peuvent tourner tantôt ainsi, tantôt autrement, parfois même auraient pu aboutir au contraire de ce qu'ils sont. Pour former et fortifier une moyenne, il faut donc que les valeurs supérieures ou particulières soient beaucoup moins fréquentes que les valeurs moyennes, qu'elles ne se présentent presque jamais, et qu'il en aille de même des valeurs anormalement basses. Au mieux, ou au pis, les unes comme les autres restent des "valeurs aux limites ", et cela non seulement dans la méthode de calcul, mais dans l'expérience, partout où règnent des conditions d'ordre arbitraire ${ }^{10}$.

11 Ce qui est en jeu dans ce passage, c'est le fondement du calcul des probabilités, c'est-àdire le comportement des événements qui est requis pour que l'on puisse en calculer une moyenne en suivant les règles de la probabilité. Plus précisément, ce fondement est double : d'une part, " les événements peuvent tourner tantôt ainsi, tantôt autrement ", d'autre part, il doit y avoir une certaine proportion entre valeurs moyennes et valeurs anormalement inférieures ou supérieures. Mais le plus important dans ce passage, mais aussi de manière plus générale dans L'Homme sans qualités, c'est que l'usage des probabilités qui intéresse Robert Musil est celui des statistiques.

12 Cette différence entre les deux auteurs, articulée à celle que l'on trouve entre conception logique et conception statistique des probabilités, demande pourtant à être nuancée. En effet, chez Ludwig Wittgenstein, on ne trouve pas seulement une définition logique de la probabilité, mais aussi une explicitation des rapports entre probabilités et fréquences, qui se révèle finalement assez proche du propos de Robert Musil. À la fin des Remarques philosophiques, deux situations sont envisagées. La première renvoie à l'impression que la fréquence observée vérifie à proprement parler le calcul de la probabilité. Or, l'impression est trompeuse dans la mesure où la fréquence observée vérifie seulement les bases de ce calcul, c'est-à-dire les lois naturelles à partir desquelles le calcul est produit :

Si je lance un dé, par exemple, je peux prédire - en apparence a priori - qu'en moyenne le chiffre 1 apparaîtra une fois sur six coups, puis je peux le confirmer par l'expérience. Mais ce que je confirme par l'expérimentation, ce n'est pas le calcul, mais la loi naturelle que le calcul des probabilités peut me présenter sous des formes différentes. En passant par le médium du calcul des probabilités, je contrôle la loi naturelle qui se trouve à la base du calcul.

Dans le cas que nous venons de voir, cette loi naturelle se représente ainsi : il y a une probabilité égale pour chacune des six faces d'être amenée au sommet du dé. C'est cette loi que nous vérifions ${ }^{11}$. 
L'autre situation est celle dans laquelle justement la fréquence observée ne correspond pas à la prédiction avancée, par exemple dans laquelle un joueur de dé produit un 1 pendant une semaine, alors que le dé n'est pourtant pas pipé et même que d'autres joueurs produisent des résultats normaux. Que conclure de cette déviation de la fréquence par rapport au calcul ? De même qu'une fréquence qui correspond au calcul ne le vérifie pas, une fréquence qui ne lui correspond pas ne l'infirme pas. La question essentielle est plutôt : ce joueur « est-il alors fondé à penser que c'est l'action d'une loi naturelle qui ne lui fait lancer que des 1 ; est-il fondé à croire que cela va continuer de la sorte, ou est-il fondé à faire la conjecture que cette régularité ne peut plus durer bien longtemps ? $»^{12}$ Autrement dit : cet écart entre la probabilité et la fréquence doit-il être attribué au hasard, auquel cas cette fréquence doit finalement converger avec le calcul, ou bien à une loi naturelle, auquel cas il est possible de changer le calcul sur la base de cette nouvelle loi, si bien que la fréquence et le calcul concordent? Selon Ludwig Wittgenstein, il est très peu probable que ce joueur accepte de reconnaître dans ses jets l'effet d'une loi : cela s'opposerait aux expériences qu'il a pu accumuler, c'est-à-dire aux fréquences observées jusque là. Mais le plus important, c'est qu'il en va dans cette situation de la nécessité de « tracer une frontière entre le hasard et la loi »" ${ }^{13}$, et que c'est la masse des fréquences observées dans le passé qui permet d'en décider.

Or, cela se rapproche assez de ce qu'expose Ulrich à Gerda, un autre personnage de L'Homme sans qualités :

Vous vous souvenez sans doute, par les cours que vous avez suivis, comment les choses se passent quand on aimerait savoir si un phénomène relève ou non d'une loi ? Ou bien on a d'avance ses raisons de le croire, comme par exemple en physique et en chimie, et même si les observations ne donnent jamais la valeur cherchée, elles n'en restent pas moins, de quelque manière, dans les parages, de sorte qu'on peut calculer cette valeur à partir d'elles. Ou bien on n'a pas ces raisons, comme c'est souvent le cas dans la vie, et on se trouve devant un phénomène dont on ne sait pas exactement s'il relève de la loi ou du hasard: alors le problème humain devient passionnant. On commence par transformer sa pile d'observations en pile de chiffres; on établit des classes (quels nombres se situent-ils entre telle ou telle valeur, entre telle valeur et la suivante, et ainsi de suite ?) et l'on en tire des lois de répartition: on constate alors que la fréquence du phénomène présente, ou ne présente pas, des variations systématiques; on obtient une distribution stationnaire, ou loi de distribution, on calcule l'écart moyen, la déviation par rapport à une valeur quelconque, l'écart médian, l'écart moyen quadratique, l'écart type, la fluctuation, et ainsi de suite, et c'est à l'aide de toutes ces notions que l'on examine le phénomène donné ${ }^{14}$.

15 Tout ce travail statistique sert à établir la fréquence et les variations d'un phénomène, pour savoir s'il relève d'une loi ou du hasard. Il ne s'agit donc ni d'identifier sa fréquence à une loi qui le gouvernerait ni même de conclure automatiquement de cette fréquence à la présence d'une loi : «[...] il y a aussi des observations qui ont toutes les apparences d'une loi naturelle sans se fonder pourtant sur quoi que ce soit que l'on puisse considérer comme telle. La régularité des séries statistiques est quelquefois aussi grande que celle des lois $»^{15}$. Au mieux, l'établissement des fréquences et des variations donne donc des raisons pour décider si le phénomène relève du hasard ou de ce qui seulement s'apparente à une loi.

16 À partir de là, on pourrait comparer plus précisément Robert Musil et Ludwig Wittgenstein sur le rôle des fréquences par rapport aux probabilités : elles servent non pas à les définir, comme si le calcul des probabilités n'était que l'expression d'une 
fréquence, mais à montrer des régularités qui peuvent être des raisons sur la base desquelles des lois peuvent être supposées et des calculs en termes de probabilités effectués. En même temps, subsistent deux différences. La première concerne la nature des fréquences observées: il s'agit, chez Ludwig Wittgenstein, de la masse des expériences antérieures, chez Robert Musil, de fréquences issues des statistiques. La seconde concerne la nature de la loi qui en est tirée : chez Ludwig Wittgenstein, on décide ou non de renvoyer une fréquence à une loi naturelle, alors que chez Robert Musil, la fréquence ne fournit au mieux qu'un analogue de loi. Au fond, subsiste la différence entre une conception statistique et une conception inductive des probabilités, même si l'enjeu est commun - tracer la frontière entre loi et hasard - et le moyen analogue - utiliser les fréquences acquises ou établies statistiquement comme des raisons pour décider de cette frontière.

Peut-on alors aller jusqu'à dire comme Georg Henrik Von Wright que « nous tendons à tracer cette frontière de façon à comprimer au maximum la marge du hasard et à étendre la portée de la loi autant que faire se peut $»^{16}$ ? On peut être réservé à l'égard de cette formule. Ce que montre l'exemple pris par Ludwig Wittgenstein, c'est la résistance du joueur de dé à "reconnaître comme une loi naturelle son incapacité à lancer autre chose que des $1 »^{17}$, et donc la résistance à modifier les lois naturelles sur lequel il fonde son calcul des probabilités. Mais comment interpréter cette résistance ? D'un côté, la portée de la loi n'est pas étendue, puisqu'il est presque certain qu'il attribuera ses jets au hasard et ne reconnaîtra pas une nouvelle loi. Mais d'un autre côté, cette résistance témoigne de son attachement à la loi naturelle présupposée dans son calcul : il y a une probabilité égale pour chacune des six faces. Autrement dit, un événement tout à fait particulier, dont on ne sait pas s'il relève du hasard ou d'une nouvelle loi, a peu de poids face à cette première loi qui provient de l'accumulation des expériences antérieures. Ainsi, nous ne modifions et n'étendons pas facilement nos lois, mais en revanche l'observation des fréquences acquiert au cours du temps un poids de plus en plus important.

De son côté, Robert Musil se montre assez prudent sur l'ampleur de l'application des statistiques aux événements historiques et intellectuels :

Une seule phrase, dans tout cela, était solide : supposé un jeu de hasard, le résultat montrerait la même répartition de chances et de malchances que la vie. Mais que le second membre de cette phrase hypothétique soit vrai ne permet nullement de conclure à la vérité du premier. Pour être croyable, la réversibilité du rapport exigerait une comparaison plus précise qui permettrait d'appliquer les notions de la probabilité aux événements historiques et intellectuels et de confronter deux domaines aussi différents ${ }^{18}$.

Que la vie et un jeu de dé offrent une répartition semblable de chances et de malchances ne permet pas d'affirmer que la vie est elle-même un jeu de hasard, si bien que la possibilité d'appliquer à la vie le calcul des probabilités, dont le jeu de dé est un modèle, dépend d'une comparaison plus précise entre la vie et le jeu de dé. De ce point de vue, les problèmes de Robert Musil sont assez différents de ceux de Ludwig Wittgenstein. Alors que ce dernier cherche seulement à articuler au mieux calcul des probabilités et fréquences, le premier cherche à savoir si le calcul des probabilités peut être appliqué à la vie et aux événements historiques et intellectuels. Or, que l'on puisse dans ce domaine utiliser les statistiques pour faire le tri entre ce qui relève d'une loi et ce qui relève du hasard, n'implique pas nécessairement que l'on puisse y appliquer le calcul des probabilités: on ne peut conclure aussi simplement d'une description 
statistique d'un phénomène à la possibilité de lui appliquer les calculs de la probabilité. Pour répondre à Georg Henrik Von Wright, on dira donc que les statistiques, sans aucun doute, voient leur portée grandir de plus en plus, mais, d'une part, que les séries statistiques ne sont pas des lois naturelles, et d'autre part, que la possibilité d'établir de telles séries ne dit encore rien de la possibilité d'appliquer le calcul des probabilités à la vie et aux événements historiques et intellectuels.

\section{De l'intérêt des probabilités pour l'invention de nouvelles possibilités}

Le sens des réflexions de Robert Musil sur la probabilité s'exprime dans le chapitre où il décrit le sens du possible. Le poids de la probabilité semble s'opposer à l'exercice de ce sens très particulier :

C'est la réalité qui éveille les possibilités, et vouloir le nier serait parfaitement absurde. Néanmoins, dans l'ensemble et en moyenne, ce seront toujours les mêmes possibilités qui se répéteront, jusqu'à ce que vienne un homme pour qui une chose réelle n'a pas plus d'importance qu'une chose pensée. C'est celui-là qui, pour la première fois, donne aux possibilités nouvelles leur sens et leur destination, c'est celui-là qui les éveille ${ }^{19}$.

1 On retrouve dans ce passage les éléments qui servent à définir la probabilité: l'adoption d'un point de vue d'ensemble attentif à la moyenne, la répétition des mêmes phénomènes et enfin la dépendance des possibilités à l'égard de la réalité. Quelle est alors exactement la position d'Ulrich face à cette répétition des mêmes possibilités ? Précisément, ce texte semble montrer qu'il ne rentre pas dans cette logique, qu'il est justement celui pour qui ce qu'impose la réalité n'a pas plus d'importance que ce que lui peut imaginer. Dans ce texte, le sens du possible s'oppose donc à la probabilité.

En même temps, dans le chapitre où il expose à Gerda la manière d'établir des séries statistiques, on peut discerner un changement d'attitude à l'égard des probabilités :

[Gerda] - Possibilités! C'est ainsi que vous pensez toujours. Jamais vous n'essaierez de vous demander comment les choses devraient être!

[Ulrich] - Vous êtes si pressés! Vous voulez toujours avoir un but, un idéal, un programme, un absolu. Et ce qui en résulte pour finir n'est jamais qu'un compromis, une moyenne ! Ne reconnaitrez-vous pas qu'il est ridicule et pénible à la longue de toujours poursuivre ou réaliser des desseins extrêmes pour n'aboutir jamais qu'à du médiocre ${ }^{20}$ ?

Même si celui qui est doué du sens du possible invente de nouvelles possibilités par rapport à ce qui s'impose de plus probable, il se démarque des partisans de «ce qui devrait être » et plus généralement des idéalistes, par sa conscience du poids du probable et de ses effets sur les injonctions et les idées. De ce point de vue, l'homme doué $\mathrm{du}$ sens du possible ne manque pas du sens des réalités, contrairement aux idéalistes. Ou plutôt, son manque de sens des réalités n'est pas «une véritable déficience $»^{21}$, comme ce peut être le cas chez les idéalistes, mais l'envers d'une "disposition créatrice $»^{22}$. Ulrich ne se fait pas d'illusion sur les possibilités qu'il crée, sur leur devenir dans la réalité.

Un troisième passage, tiré d'un chapitre déjà cité, envisage même la fécondité de cette vision probabiliste du monde :

Agathe se demanda si confondre le train du monde et le hasard n'était pas une

façon capricieuse de noircir la vérité, l'effet d'un pessimisme romantique. 
«Pas le moins du monde! répliqua Ulrich. Nous sommes partis de la vanité de toutes les nobles espérances, et nous avons cru y découvrir un perfide mystère. Mais si nous la confrontons maintenant avec les règles de la probabilité, nous expliquerons fort modestement ce mystère [...]. Du même coup, à partir du probable, nous expliquerons le règne, la stabilité, l'accroissement fort indésirable de tout ce qui est moyen! Rien là de romantique, ni même peut-être de noir ! Qu'on le veuille ou non, ce serait plutôt une tentative courageuse ! ${ }^{23}$

L'intérêt de ce type d'application, dont la dimension pourtant problématique sera soulignée quelques lignes plus loin, est explicitement de nature théorique: ce qui apparaît comme un mystère, la vanité des idées et des espérances face à la réalité, devient tout à fait explicable par l'application des règles de la probabilité. Mais on peut aussi imaginer que cet intérêt soit, chez cet ancien ingénieur qu'est Ulrich, de nature pratique : pouvoir expliquer le probable, et notamment l'état moyen dans lequel nous vivons et les hommes moyens que nous sommes, c'est acquérir une emprise sur lui. De ce point de vue, Ulrich ne fait qu'exprimer un trait caractéristique du XIX ${ }^{e}$, que Ian Hacking a appelé la domestication du hasard ${ }^{24}$.

Il n'en reste pas moins que le sens du possible se définit comme une capacité à créer de nouvelles possibilités, distincte du travail statistique et du calcul des probabilités. Or, là encore, aussi bien chez Robert Musil que chez Ludwig Wittgenstein, les mathématiques jouent un rôle essentiel dans cette création.

L'essai de Robert Musil intitulé «L'homme mathématique» est capital: l'auteur y déploie progressivement l'importance non seulement de la place des mathématiques mais en plus de leur créativité. Cela se montre dès le deuxième paragraphe où il se démarque de ce qui ressemble fortement à une idée d'Ernst Mach :

On dit qu'elles [les mathématiques] représentent pour la pensée le maximum d'économie, et sans doute est-ce exact. Mais le fait même de penser est une affaire obscure et problématique. C'est devenu depuis longtemps (quand même ç'aurait été d'abord une simple épargne biologique) une complexe passion d'épargner qui ne se soucie pas plus de l'ajournement du résultat que l'avare de sa pauvreté lentement, voluptueusement, convertie en son contraire ${ }^{25}$.

作 comme recherche du «maximum d'économie» et de la pensée comme "épargne biologique ». Mais ce qui est montré, c'est que leur dimension passionnelle leur a fait oublier leur finalité, de sorte que la pensée et les mathématiques sont devenues des activités en quelque sorte autonomes et donc libres.

Cette ambivalence se retrouve alors dans la suite de cet essai. Dans un premier temps, c'est l'aspect « économique » des mathématiques qui est développé, avec notamment l'exemple des machines à calculer. Là où sans doute nous aurions tendance à en condamner l'usage au nom de la paresse qu'elles engendrent, Robert Musil met en valeur la rapidité, l'efficacité et la puissance de l'instrument. Mais le plus important, c'est que les mathématiques elles-mêmes sont pensées sur le modèle de la machine à calculer : « on peut considérer les mathématiques comme un appareil intellectuel idéal dont le but, et le succès, sont de prévoir, à partir des principes, tous les cas possibles ${ }^{26}$. Elles semblent donc mêler à la fois une dimension pratique et économique, et une grande puissance : prévoir tous les cas possibles. De ce point de vue, les mathématiques n'ont pas pour seul rôle important de mesurer la probabilité des événements, mais aussi d'envisager tous les cas possibles, quelle qu'en soit la probabilité. 

dimension créatrice des mathématiques, puisque ces possibilités restent liées à des fins pratiques : «Il faut donc détourner son regard des profits extrinsèques, l'appliquer, à l'intérieur même des mathématiques, à la répartition des éléments restés inutilisés, pour découvrir l'autre visage de cette science. Alors, rien moins qu'efficace, elle se révèle de nature dispendieuse et passionnelle $»^{27}$. Ce qu'il cherche à mettre en valeur, c'est justement le contraste entre les quelques parties des mathématiques qui sont utilisées et tout le reste qui ne l'est pas. L'homme moyen, l'ingénieur et même le physicien n'utilisent des mathématiques que peu de choses, alors que "tout à côté, s'étendent d'immenses domaines qui n'ont d'existence que pour le mathématicien ${ }^{28}$. L'usage des mathématiques est donc loin de se cantonner à la mesure de la probabilité et à la prévision des différents cas possibles, toutes deux limitées par leur rapport pratique au réel, mais se prolonge dans une création prodigue de nouvelles possibilités.

Que les mathématiques soient créatrices de nouvelles possibilités est une idée que l'on trouve aussi chez Ludwig Wittgenstein, mais exprimée de manière beaucoup plus radicale. En effet, que les mathématiques prévoient tous les cas possibles ou que le mathématicien s'occupe d'un domaine qui lui est propre, à côté de la réalité ordinaire, sont deux expressions qu'il aurait cherché à remettre en cause - à supposer que ces expressions doivent être combattues dans ce contexte d'expression. Elles ne devraient l'être en effet que dans la mesure où elles orientent une théorisation philosophique, mais pas nécessairement dans un autre contexte d'énonciation, par exemple celui d'essais destinés à être publiés dans des journaux.

En tout cas, ce qu'il peut y avoir de contestable d'un point de vue philosophique, c'est l'idée qu'il y aurait des possibilités à prévoir et à explorer, qui préexisteraient à ces deux opérations et constitueraient ce que Jacques Bouveresse a appelé en référence à Leibniz un "pays des possibles $»^{29}$. Cela suppose selon Ludwig Wittgenstein que, paradoxalement, les possibilités forment une autre réalité ou une réalité d'un autre type, à côté de la réalité au sens ordinaire :

Frege, qui était un grand penseur, a soutenu que, bien qu'Euclide ait dit qu'il était possible de tirer une droite entre deux points quelconques, en réalité la droite existait déjà, même si personne ne l'avait jamais tirée. L'idée est qu'il y a un royaume géométrique dans lequel existent les entités géométriques. Ce que nous nommons, dans le monde quotidien, une possibilité est, dans le monde géométrique, une réalités ${ }^{30}$.

Cela a alors deux conséquences. D'une part, la possibilité est considérée comme "l'ombre de la réalité ", dans la mesure où l'on suppose derrière chaque point, chaque ligne, chaque figure, une possibilité de nature idéale, qui en est comme l'ombre ontologique. D'autre part, les mathématiques pures sont considérées comme une description: les mathématiques pures comme description de telles entités à la fois possibles et idéales, les mathématiques appliquées comme description des figures et des nombres concrets. Or, sur ce dernier point, ce qui pose problème aux yeux de Ludwig Wittgenstein, ce n'est pas tant l'objet de la description, qu'il soit idéal ou empirique, mais la description elle-même. Les mathématiques ne sont tout simplement pas une description, mais opèrent d'une manière toute différente.

Cela se voit notamment à son traitement de l'impossibilité mathématique, par exemple l'impossibilité de la construction d'un heptagone au moyen d'une règle et d'un compas :

La preuve mathématique qui établit l'impossibilité de construire un heptagone

régulier à la règle et au compas a pour seul effet de nous donner de bonnes raisons

Philonsorbonne, 5 | 2011 
d'exclure de notre notation l'expression « construction de l'heptagone ». D'où le fait que la proposition: "Smith a tracé la construction de l'heptagone » n'est pas fausse, mais dépourvue de sens. Elle fait appel à une expression à laquelle non seulement on n'a donné aucun sens mais qui, de surcroît, a été exclue ${ }^{31}$.

On pourrait croire qu'établir l'impossibilité d'une telle construction, c'est d'abord penser à sa possibilité puis en montrer la fausseté et l'interdire. En réalité, une démonstration mathématique a un rôle bien plus radical: elle détermine ce qui est possible et ce qui ne l'est pas. Autrement dit, le possible et l'impossible ne sont pas le point de départ de la démonstration, mais son résultat. Établir une possibilité ou une impossibilité en mathématiques, ce n'est pas tant établir un fait, qu'établir une règle. On comprend alors en quel sens Ludwig Wittgenstein soutient l'idée que les mathématiques créent de nouvelles possibilités: elles inventent des règles qui déterminent ce qui est possible et ce qui ne l'est pas, qui établissent ce qui a du sens et ce qui n'en a pas.

On terminera en opérant un rapprochement entre ce qui vient d'être dit des mathématiques et ce que Robert Musil dit de la littérature. À ses yeux, les mathématiques sont un modèle pour la culture, comme le développe la fin de «L'homme mathématique ", mais aussi pour la littérature, comme le développe cette fois un autre essai essentiel, «La connaissance de l'écrivain : esquisse » :

Telle est la patrie de l'écrivain, où sa raison est suzeraine. Alors que son contraire cherche le solide et s'estime satisfait chaque fois qu'il peut établir, dans ses calculs, autant d'équations qu'il découvre d'inconnues, ici, de prime abord, il n'y a pas de limites aux inconnues, aux équations, aux possibilités de solution. La tâche consiste à découvrir sans cesse de nouvelles solutions, de nouvelles constellations, de nouvelles variables, à établir des prototypes de déroulement d'événements, des images séduisantes des possibilités d'être un homme, d'inventer l'homme intérieur ${ }^{32}$.

Ce passage peut donner l'impression de réserver à la littérature l'invention de nouvelles possibilités, les mathématiques étant davantage limitées en termes de solution à trouver dans leur domaine. En réalité, les secondes offrent un bon point de comparaison pour penser la tâche de la première.

abord, elles permettent de penser la nature des problèmes littéraires. Dans ce passage, Robert Musil semble en effet réutiliser ce qu'il a dû lire chez Louis Couturat à propos des mathématiques chez Leibniz:

Quand on a autant d'équations (indépendantes) que d'inconnues à trouver, le problème est déterminé, et comporte une ou plusieurs solutions. Quand on a moins d'équations que d'inconnues, le problème devient indéterminé, et comporte une infinité de solutions. Enfin, quand il y a plus d'équations que d'inconnues, il faut que ces équations soient compatibles entre elles, sinon le problème devient impossible $e^{33}[. .$.

Dans cette échelle des différents degrés de détermination des problèmes algébriques, la littérature semble avoir à faire à des problèmes qui s'intercalent entre les problèmes indéterminés et les problèmes impossibles. Ce que les problèmes littéraires ont en commun avec les problèmes indéterminés, c'est l'infinité de solutions, qui correspond en littérature à l'infinité des issues possibles à ce qui est raconté. Mais ce qui les différencie de ces problèmes indéterminés, c'est l'infinité des équations, c'est-à-dire des scénarii possibles. Les mathématiques permettent donc de penser par analogie l'indétermination radicale des problèmes littéraires. 
En même temps, toujours à titre de point de comparaison, elles permettent de penser la spécificité de la solution littéraire à ces problèmes. Il s'agit pour la littérature d'établir, d'inventer de nouvelles possibilités, mais de manière assez différente des mathématiques. Elle n'a pas en effet à déterminer ce qui est possible et ce qui ne l'est pas, à établir ce qui a du sens ou ce qui n'en a pas, au moyen d'une démonstration utilisant des règles et aboutissant à une nouvelle règle. Elle ne détermine ni ne règle, mais invente simplement de nouvelles " possibilités d'être un homme».

\section{NOTES}

1. R. Musil, L'Homme sans qualités, Paris, Le Seuil, 1956, tr. P. Jaccottet, t. 1, p. 20.

2. J. Bouveresse, Robert Musil. L'homme probable, le hasard, la moyenne et l'escargot de l'histoire, Paris, L'Éclat, 1993.

3. Id., La force de la règle. Wittgenstein et l'invention de la nécessité, Paris, Minuit, 1987 ; Le pays des possibles. Wittgenstein, les mathématiques et le monde réel, Paris, Minuit, 1988.

4. . H. Von Wright, Wittgenstein, Mauvezin, TER, 1986, tr. É. Rigal, p. 155.

5. R. Musil, Journaux, Paris, Le Seuil, 1981, t. 1, p. 557-568.

6. H. E. Timerding, Die Analyse des Zufalls, Braunschweig, Vieweg \& Sohn, 1915.

7. 7. F. Waismann, "Logische Analyse des Wahrscheinlichkeitsbegriffs", Erkenntnis, 1 (1930/1931), notre traduction, p. 228.

8. L. Wittgenstein, Tractatus logico-philosophicus, Paris, Gallimard, 1993, trad. fr. Gilles-Gaston Granger, p. 74.

9. Ibid., p. 74-76.

10. R. Musil, L'Homme sans qualités, Paris, Le Seuil, 1956, t. 2, trad. fr. P. Jaccottet, p. 509.

11. L. Wittgenstein, Remarques philosophiques, Paris, Gallimard, 1975, trad. fr. J. Fauve, p. 276.

12. Ibid., p. 278.

13. G. H. Von Wright, op. cit., p. 169.

14. R. Musil, op. cit., t. 1, p. 613.

15. Ibid., p. 614.

16. G. H. Von Wright, op. cit., p. 169.

17. L. Wittgenstein, op. cit., p. 278.

18. Robert Musil, op. cit., t. 2, p. 511.

19. R. Musil, op. cit., t. 1, p. 21.

20. Ibid., p. 616.

21. Ibid., p. 20.

22. Ibid.

23. R. Musil, op. cit., p. 510.

24. I. Hacking, The Taming of Chance, Cambridge, Cambridge University Press, 2000.

25. R. Musil, Essais, Paris, Le Seuil, 1978, tr. P. Jaccottet, p. 56.

26. Ibid., p. 56-57.

27. Ibid., p. 57.

28. Ibid.

29. Cf. n. 3 : ouvrage du même nom. 
30. L. Wittgenstein, Cours sur les fondements des mathématiques. Cambridge 1939, traduction É. Rigal, Mauvezin, TER, 1995, p. 144.

31. Ibid., p. 37.

32. Ibid., p. 83.

33. L. Couturat, La logique de Leibniz, Hildesheim - Zurich - New York, Georg Olms Verlag, 1985, p. 252-253. Louis Couturat souligne. Son nom est cité plusieurs fois dans les Journaux de Musil.

\section{RÉSUMÉS}

Il y a une parenté entre Musil et Wittgenstein dans le rôle central qu'ils accordent à la notion de possibilité. Cet article analyse plus précisément le lien essentiel qu'ils instaurent entre cette notion et les mathématiques: en quoi les probabilités peuvent-elles être définies comme une mesure des possibilités? Quel est notamment le rapport entre définition fréquentiste et définition logique des probabilités? En quel sens peut-on dire, par ailleurs, que les mathématiques sont un modèle d'invention de nouvelles possibilités?

\section{INDEX}

Mots-clés : Musil, Wittgenstein, possibilité, probabilité, statistiques, fréquence, calcul, moi, hasard, mathématiques, réalité, impossibilité, littérature, détermination 